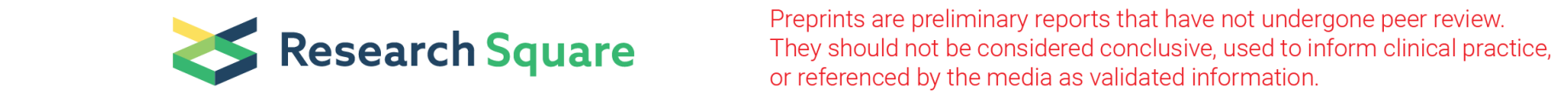

\title{
Long -Term Persistence of Antibodies Against Varicella in Fully Immunized Healthcare Workers: An Italian Retrospective Cohort Study
}

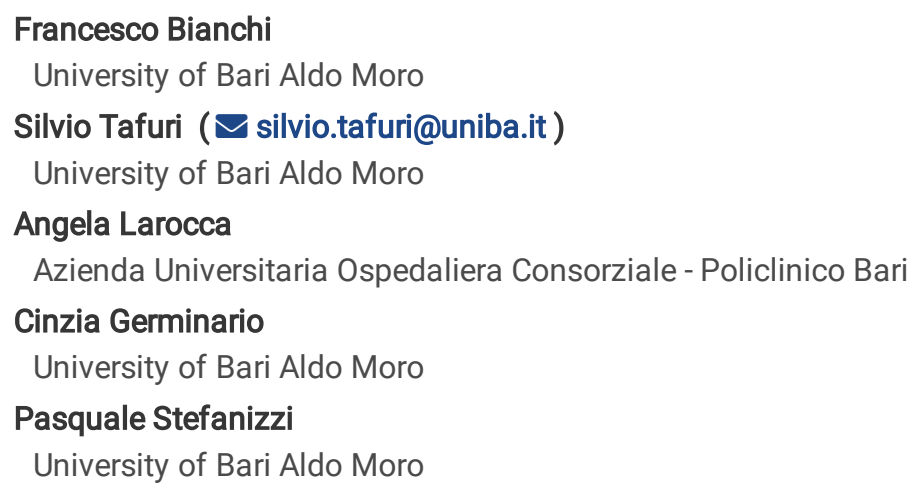

Keywords: Varicella zoster virus, third Varicella dose, duration of immunization, healthcare workers, long-term immunogenicity, chickenpox Posted Date: January 20th, 2021

DOI: https://doi.org/10.21203/rs.3.rs-142042/v1

License: @ (i) This work is licensed under a Creative Commons Attribution 4.0 International License. Read Full License 


\section{Abstract}

Background. Chickenpox is a highly contagious disease caused by the varicella zoster virus (VZV), and in infants, adolescents, adults, pregnant women, and the immunocompromised it can be serious. The best way to prevent chickenpox is immunization with the varicella vaccine. Protective levels of antibodies induced by the varicella vaccine decline over time, but there is currently no formal recommendation for testing anti-varicella zoster virus (VZV) IgG levels in immunized healthcare workers (HCWs).

Methods. The aims of this study were to evaluate the seroprevalence of circulating anti-VZV IgG in a sample a sample of students and residents of the medical school of the University of Bari, the long-term immunogenicity of the varicella vaccine, and the effectiveness of a strategy consisting of a third vaccine booster dose. The study population was screened as part of a biological risk assessment conducted between April 2014 and October 2020. A strategy for the management of non-responders was also examined.

Results. The 182 students and residents included in the study had a documented history of immunization (two doses of varicella vaccine). The absence of anti-VZV IgG was determined in $34 \%(62 / 182 ; 95 \% \mathrm{Cl}=27.2-41.4 \%)$, with serosusceptibility more common among males than females $(\mathrm{p}<0.05)$. After a third varicella dose, seroconversion was achieved in $100 \%$ of this previously seronegative group. No serious adverse events were recorded.

Conclusions. One-third of the study population immunized against VZV lacked a protective antibody titer, but a third dose of vaccine restored protection. Since it is highly unlikely that VZV will be eliminated in the immediate future, the loss of immunity in a substantial portion of the population implies a risk of varicella outbreaks in the coming years. Screening for varicella immunity in routine assessments of the biological risk of medical students and HCWs may help to prevent nosocomial VZV infections.

\section{Background}

Chickenpox is a highly contagious disease caused by the varicella zoster virus (VZV), and in infants, adolescents, adults, pregnant women, and the immunocompromised it can be serious [1]. VZV causes a systemic infection that typically results in lifetime immunity [2] but endogenous reactivation from latency can cause herpes zoster (HZ), commonly known as "shingles" [3]. The best way to prevent chickenpox is immunization with the varicella vaccine, available both in monovalent form and in combination with the measles, mumps and rubella (MMRV) vaccine [4]. In the US, since the introduction of the vaccine, in 1995, > 3.5 million cases of varicella, 9,000 hospitalizations, and 100 deaths have been prevented [5]. Furthermore, between 1993 and 2013 there was a 17\% decline in the rate of $\mathrm{HZ}$ among children, coinciding with the introduction of routine varicella vaccination [6].

At the time of the writing of this study, CDC provides that youngsters receive two doses of varicella vaccine, specifically the first one at 1215 months and the second at 4-6 years [7]. Moreover, not immunized teenagers and adults should get two doses of the MMRV/varicella vaccine from 4 to 8 weeks apart [7].

Pre-licensure data show that one dose of vaccine prevent any manifestation of varicella in $82 \%$ of cases and $100 \%$ successful against the most serious complications of the disease; two doses prevent any manifestation of varicella in $98 \%$ of cases and $100 \%$ successful against the most serious complications of the disease [8]. Post-licensure evidences show that two doses of vaccine are $92 \%$ effective versus all manifestations of disease [8]. The seroconversion rate after two doses is estimated to be $>95 \%$ [2].

Since the introduction of global mass vaccination, the varicella vaccine has shown a high level of safety, cost-savings, and efficacy [2, 9, 10]. However, according to the $C D C$, the duration of protection against varicella following vaccination is unclear. Live vaccines generally provide long-lasting immunity, but thus far only few studies have shown that vaccination against varicella confers protection for almost 10 years thereafter [8].

Since 2003, Italy's National Immunization Plan has recommended two doses of varicella vaccine, in accordance with CDC guidelines [7]. In 2017, varicella vaccination became compulsory in Italy, mandated by the Italian Ministry of Health, Decree-Law n. $73 / 2017$ [11]. Currently, the Italian National Immunization Plan recommends the administration of either the monovalent varicella vaccine or the tetravalent MMRV vaccine [12]. Despite these measures, the proportion of susceptible Italian adults remains high. For example, in two very recent studies of Italian healthcare workers (HCWs), including those whose vaccination status was unknown, VZV susceptibility was in the range of 6.7$12 \%[13,14]$.

The aims of this study were to evaluate the seroprevalence of circulating anti-VZV IgG in a sample of young adult HCWs, the long-term immunogenicity of the varicella vaccine, and the effectiveness of a strategy consisting of a third vaccine booster dose administered to previously immunized adults without detectable IgG against VZV. The study was carried out in Apulia (southern Italy, $4,000,000$

Page $2 / 9$ 
inhabitants), a region where previous surveys found a relatively high (7-12\%) prevalence of adults susceptible to VZV [15, 16] and where varicella outbreaks have been recorded [17].

\section{Methods}

Since 2010, the Italian Ministry of Health has required that medical schools and university hospitals apply the same procedures mandated by Italian law for the occupational health and safety of HCWs to medical students and residents as well [18]. In response, in April 2014 the Hygiene Department of the Bari Policlinico University Hospital implemented a biological risk prevention program for students and residents of the medical school of the University of Bari. As part of the program, VZV susceptibility/immunity was assessed.

This study was designed as a retrospective cohort study with a study population comprising Apulian students and residents of the medical school of the University of Bari who underwent a biological risk assessment between April 2014 and October 2020 . The study exclusion criteria were absence of an available vaccination history, no history of vaccination or vaccinated with a single dose or $\geq 3$ doses of varicella vaccine at baseline, and a history of varicella infection. Inclusion criteria were vaccination with two doses of varicella vaccine (vaccine basal routine), either monovalent or tetravalent, prior to study enrollment.

The vaccination status of each participant was confirmed using the Regional Immunization Database (GIAVA) [18], a computerized vaccination registry that contains the vaccination history of every Apulian inhabitant. A 5-mL serum sample was collected from each enrollee to assess his/her VZV immunity/susceptibility status and was tested by chemiluminescence using LIAISONß VZV IgG, a semiquantitative method performed with a standardized commercial kit (Diasorin). An anti-varicella lgG titer $>150 \mathrm{mIU} / \mathrm{mL}$ is defined as seroprotective [19].

Tested participants with a non-protective IgG titer received a third booster dose of monovalent varicella vaccine, administered subcutaneously in the deltoid muscle, consistent with the protocols applied in some US medical schools [20]. Participants with equivocal tests were retested and, if the result was still equivocal, they were classified as seronegative. A further blood test was performed $20-25$ days after vaccination to re-measure IgG titers; a value that exceeded the cut-off was considered to indicate seroconversion. If the titer was still negative, another vaccine dose (28 days after the first booster) was administered and after another 20-25 days the IgG titer was again measured. Students/residents who were still seronegative were definitively classified as "non-responders." Anyone who received a booster dose was followed-up for 1-month to monitor the possible occurrence of adverse effects.

Information on patient identification, sex, age at study enrollment, dates of routine varicella vaccine administration, VZV IgG titer, date of first booster dose, IgG titer after the first booster, date of the second booster dose, and IgG titer after the second booster were collected. The data were imputed in a database created using Office Excel software and analyzed using STATA MP16 software [21].

Continuous variables are expressed as the mean \pm standard deviation and range, categorical variables as proportions, with the $95 \%$ confidence interval $(95 \% \mathrm{Cl})$, when appropriate. Skewness and kurtosis tests were used to evaluate the normality of the continuous variables, but any of them were normally distributed or normalizable. The Wilcoxon's rank sum test (not parametric) was used to compare continuous variables between the sexes and the Wilcoxon's signed-rank test (not parametric) was used to compare them as a function of the evaluation time. Chi-squared and Fisher's exact tests were used to compare proportions [21].

The determinants of seropositivity at enrollment were assessed in a multivariate logistic regression analysis that included sex (male vs. female), age at enrollment (years), the presence of one or more chronic diseases (yes/no), age at the time of the first vaccination of the basal routine (years), and age at the time of the second vaccination of the basal routine (years). The adjusted odds ratio (aOR) was calculated with the $95 \% \mathrm{Cl}$. The Hosmer-Lemeshow test was used to evaluate the goodness-of-fit of the multivariate logistic regression model [21].

Protective antibody survival (PAS), defined as the time from the second dose of routine varicella vaccine until the evaluation of the antibody titer, was evaluated using Kaplan-Meier curves, and the differences between sexes using the log-rank test. The median PAS time was estimated and the incidence of the loss of seroprotection per person-year was calculated together with the incidence rate ratio (IRR). In the latter, the incidence in females served as the denominator and that in males as the numerator.

A multivariate Cox semiparametric regression was used to evaluate the determinants of PAS; the risk predictors were as follows: sex (male vs. female), age at enrollment (years), presence of one or more chronic diseases (yes/no), age at the first dose of the routine vaccine (years), and age at the second dose of the routine vaccine (years). The adjusted hazard ratio (aHR) was calculated along with the $95 \% \mathrm{Cl}$. The Schoenfeld and scaled Schoenfeld residuals tests were used to evaluate the proportionality assumption of the multivariate Cox 
semiparametric regression model; if one of the predictors was not proportional, the regression model was stratified on the non-proportional predictor. The Gronnesby and Borgan test was used to evaluate the goodness-of-fit of the model.

For all tests, a two-sided p-value $<0.05$ was considered to indicate statistical significance.

\section{Results}

From April 2014 to October 2020, 6,105 medical students and residents participated in the biological risk assessment program. According to the vaccination certificates, available for 5,469/6,105 (89.6\%) participants, 182/5,469 (3.3\%) received two doses of anti-varicella vaccine. Among the latter, $124(68.1 \%)$ were female; the average age at enrollment was $20.4 \pm 2.5$ years (range $=18.0-38.0)$ and did not significantly differ between females $(20.6 \pm 2.7$; range $=18.0-38.0)$ and males $(20.2 \pm 2.1$; range $=18.0-26.0 ; p=0.194)$. At least one chronic disease was reported in 68 of the 182 (37.4\%) participants, without a difference between sexes (females: 47/124; 37.9\%; males: $21 / 58 ; 36.2 \% ; p=0.826)$. None of the participants had a history of varicella.

The 182 study enrollees with a complete basal vaccination routine (two doses) were tested for their titers of anti-varicella IgG. A protective titer was determined in over half $(120 / 182 ; 65.9 \% ; 95 \% \mathrm{Cl}=58.6-72.9 \%)$ and the difference between females $(\mathrm{n}=91 / 124 ; 73.4 \% ; 95 \% \mathrm{Cl}=$ $64.7-80.9 \%)$ and males $(n=29 / 58 ; 50.0 \% ; 95 \% C l=36.6-63.4 \%)$ was statistically significant $(p=0.002)$. The overall geometric mean antivaricella IgG titer was $304.5(95 \% \mathrm{Cl}=250.1-370.8)$ and also differed significantly between females $(367.6 ; 95 \% \mathrm{Cl}=292.8-461.5)$ and males $(203.7 ; 95 \% \mathrm{Cl}=140.6-295.0 ; \mathrm{p}=0.006)$. The geometric mean neutralization titer $(\mathrm{GMT})$ among the seroprotected was $657.1(95 \% \mathrm{Cl}$ $=556.9-775.4)$, compared with $68.7(95 \% \mathrm{Cl}=59.4-79.5)$ in the non-seroprotected $(p<0.0001)$.

A booster dose was administered to 44 of the 62 (71.0\%) seronegatives. Within this group 34 (77.3\%) were re-evaluated and all (100.0\%; $95 \% \mathrm{Cl}=89.7-100.0 \%$ ) were found to have seroconverted, obviating the need for a second booster dose after study enrollment. The GMT in the seronegative group after one booster dose administered subsequent to enrollment increased from $66.9(95 \% \mathrm{Cl}=56.4-79.3)$ to $1,607.6(95 \% \mathrm{Cl}=1,253.6-2,061.7 ; \mathrm{p}<0.0001)$.

The multivariate logistic regression model showed an association of seropositivity at enrollment with male sex $(a O R=0.4 ; 95 \% \mathrm{Cl}=0.2-$ $0.7)$ and with age (in years) at the time of the first dose of varicella vaccine $(a O R=0.84 ; 95 \% \mathrm{Cl}=0.74-0.95)$. There were no associations with any of the other determinants $(p>0.05$; Table 1$)$.

Table 1

Determinants of seropositivity at study enrollment according to a multivariate logistic regression model

\begin{tabular}{|llll|}
\hline Determinant & aOR & $95 \% \mathrm{Cl}$ & p-value \\
\hline Age at enrollment (years) & 0.99 & $0.82-1.19$ & 0.904 \\
\hline Sex (male vs. female) & 0.36 & $0.28-0.72$ & 0.003 \\
\hline Chronic disease (yes $/$ no) & 0.86 & $0.44-1.68$ & 0.650 \\
\hline Age at the time of the first vaccine dose, basal routine & 0.84 & $0.74-0.95$ & 0.006 \\
\hline Age at the time of the second vaccine dose, basal routine & 1.13 & $0.95-1.33$ & 0.157 \\
\hline
\end{tabular}

Hosmer-Lemeshow $X^{2}=5.8 ; p=0.675$

The average PAS time was $5.7 \pm 2.4$ years (range $=1.0-13.0$ ), with an estimated loss of anti-varicella IgG in $50 \%$ of the study group after 9 years $(95 \% \mathrm{Cl}=8-9)$. The incidence rate of seronegativity $\times 100$ person-years was $6.0(95 \% \mathrm{Cl}=4.6-7.6)$. The difference in the estimated PAS between males and females was significant $(p=0.021$; Fig. 1$)$, with an incidence rate of seronegativity $\times 100$ person-years of 8.3 $(95 \% \mathrm{Cl}=5.8-12.0)$ and $4.8(95 \% \mathrm{Cl}=3.4-6.7)$, respectively. The IRR was $1.75(95 \% \mathrm{Cl}=1.02-2.97 ; \mathrm{p}=0.031)$.

According to the multivariate Cox semiparametric regression model, male sex ( $\mathrm{aHR}=1.73 ; 95 \% \mathrm{Cl}=1.04-2.88$ ), older age at enrollment $(\mathrm{aHR}=0.71 ; 95 \% \mathrm{Cl}=0.60-0.83)$, and older age at the time of the first vaccine dose of the basal routine $(\mathrm{aHR}=1.5 ; 95 \% \mathrm{Cl}=1.2-1.7)$ were risk factors for PAS (Table 2). 
Table 2

Analysis of risk predictors of PAS in a multivariate semiparametric Cox regression model

\begin{tabular}{|llll|}
\hline Predictor & aOR & $95 \% \mathrm{Cl}$ & p-value \\
\hline Age at enrollment (years) & 0.71 & $0.60-0.83$ & $<0.0001$ \\
\hline Sex (male vs. female) & 1.73 & $1.04-2.88$ & 0.034 \\
\hline Chronic disease (yes/no) & 0.97 & $0.58-1.62$ & 0.897 \\
\hline Age at the time of the first vaccine dose, basal routine & 1.46 & $1.27-1.67$ & $<0.0001$ \\
\hline
\end{tabular}

Groennesby and Borgan $X^{2}=1.9 ; \mathrm{p}$-value $=0.165$

An assessment of the safety of the booster dose based on a 1-month follow-up did not reveal any serious and/or long-term adverse reactions. The most commonly reported reactions were pain at the injection site $(9 \times 100$ subjects), mild fever within $10-15$ days after booster administration ( $3 \times 100$ subjects), and laterocervical lymphadenopathy ( $1 \times 100$ subjects). All of the adverse events regressed after a few days, without sequelae.

\section{Discussion}

Among the $>6,000$ young adults initially assessed in our study, only $3 \%$ had received two doses of the varicella vaccine prior to study enrollment. Since 2003, the National Immunization Plan has supported varicella vaccination for adolescents who are not naturally immunized. Thus, for the population born before 1998 , as was the case in the majority of our study participants, varicella coverage in Italy is very low.

Despite two doses of varicella vaccine, almost $34 \%(95 \% \mathrm{Cl}=27-41)$ of the enrollees did not have circulating anti-VZV IgG. However, among those who then received a booster dose, an immunity response was elicited such that the seroconversion rate was $100 \%$. Furthermore, the increase in the GMT (from 67 to 1,608) after the booster dose resulted in a significantly higher titer than in the group that was seroprotected at enrollment $(1,608$ vs. $657 ; p<0.0001)$, indicating a decrease over time in the antibody titer among the vaccinated population.

Our study showed a better immunological response and duration of circulating antibodies in females than in males, based on comparisons of seroprevalence at enrollment, GMT, and PAS, the IRR, and the multivariate regression models. Previous studies of the sexbased differences in the response to vaccines or infection $[22,23]$ have consistently shown that females have a more effective immune response to immunization, and thus presumably varicella vaccination (immunogenicity and, probably, effectiveness), and against infection.

Our antibody survival model showed that antibody levels tend to decline as early as 1 year after completion of the basal routine, such that after 9 years half of the fully vaccinated population will have lost circulating antibodies. In another study, albeit of low scientific evidence, a period of 10 years was determined [8]. For other live-attenuated virus vaccines, such as the MMR (measles, mumps, rubella) vaccine, the duration of circulating antibodies is more than double that of the varicella vaccine [21, 24, 25]. A loss of circulating antibodies over time was also shown in this study by the multivariate regression models, in which the interval between the last vaccine dose and the serological assessment was identified as a determinant of serosensitivity.

The strengths of this study are that it provides further knowledge on a topic that has been poorly studied thus far, including evidence of sex-based difference in VZV immunity. However, a major limitation was the sample size, although this was not unexpected because, in Italy, varicella vaccination for adolescents who are not naturally immunized became routine only in 2003 whereas the study participants were for the most part born before 1998. Another limitation was our inability to analyze the immune status as a function of vaccine formulation (monovalent vs. MMRV). A determination of the differences between these vaccines is crucial to understanding their immunogenicity performance. In addition, whether the study enrollees had ever come into contact with the wild virus (without developing disease) was unknown. Future research should assess the management of non-responders, which will require a larger sample size and a longer follow-up after routine vaccination, perhaps also stratifying the study population per vaccine type (monovalent vs. MMRV). This approach will provide a fuller picture of immunogenicity over time.

The efficacy of at least one (and potentially a second) booster dose in fully vaccinated but non-seroprotected healthcare workers (HCWs), such as our study population, has not been well studied. Behrman et al. [26] evaluated the humoral immune responses of $101 \mathrm{HCWs}$ 
(median age 30 years) who had previously received a two-dose regimen of varicella vaccine. including 12 (11.9\%) who were seronegative at enrollment but received a third vaccine dose during the study. Seven of those $12 \mathrm{HCWs} \mathrm{(58.3 \% )} \mathrm{seroconverted} \mathrm{after} \mathrm{the} \mathrm{third} \mathrm{dose}$ (safety data were not reported). Our study population comprised a much larger number of serosusceptibles and the seroconversion rate after the booster dose was nearly twice as high. In a 2020 Italian study, Trevisan A et al. [27] tested 234 full vaccinated medical school students and determined a serosusceptibility rate of $21.4 \%$. The authors found a decrease in the antibody titer with an increasing interval from vaccination to the antibody titer evaluation. Within the group of serosusceptible students who received a third vaccine dose, $91.4 \%$ seroconverted, without any major symptoms following vaccination. The results of that study were in agreement with our own, especially considering the similar study populations, but Trevisan A et al. [27] found no evidence of a difference between sexes.

The risk of a loss of antibodies in vaccinated individuals over time is an important finding, as between now and the next 10-15 years the loss of circulating antibodies against VZV in the vaccinated population will increase their susceptibility to the virus. Since it is highly unlikely that VZV will be eliminated in the immediate future, the loss of immunity in a substantial portion of the population implies a risk of varicella outbreaks in the coming years. Indeed, many cases of chickenpox among fully vaccinated adults have been reported in the literature, although the disease in this group seems to be less severe [28-30]. However, it should also be noted that the role of cellmediated immunity in the protection of the non-seroprotected is a matter of debate within the scientific community [26, 31], such that whether serum IgG titers alone accurately reflect vaccine protection is unclear. A 2020 study [32] compared humoral and T cell immunity in women of childbearing age who had been immunized against VZV either by vaccination or naturally. The authors found no significant differences in the antibody titers between the two groups ( $p>0.050)$, although the naturally immunized group had significantly higher levels of VZV antigen-specific CD4 T cells $(p=0.004)$.

\section{Conclusions}

Finally, while further research is needed, the results of our study have important implications for HCWs, as the inclusion of a screening model in the routine assessment of their biological risk may prevent nosocomial varicella infections, especially in high-risk settings (pediatric wards, gynecology units, infectious disease departments, etc.). Moreover, as chickenpox can be particularly severe in adults, $\mathrm{HCWs}$ should be protected by varicella vaccination, which will also confer protection from $\mathrm{HZ}$ [33].

In summary, the time between varicella vaccination and the antibody titer evaluation is a determinant of a decline in serum levels of circulating antibodies against VZV and thus of immunity to the virus. Among the fully vaccinated but non-seroprotected, the administration of a third booster dose is effective and safe in achieving seroconversion. These results are an important contribution to public health planning.

\section{Abbreviations}

CDC: Center for Disease Control and Prevention

WHO: World Health Organization

VZV: Varicella zoster virus

MMRV: measles, mumps, rubella, varicella

HCWs: healthcare workers

HZ: herpes zoster

GIAVA: Regional Immunization Database

\section{Declarations}

Ethics approval and consent to participate: The ethical committee of Osservatorio Epidemiologico Regionale approved this study. The study follows the principles of the World Medical Association Declaration of Helsinki, and does not involve any experiments on humans or human samples, nor research on identifiable human material and data. Written informed consent to participate in this study was obtained from the participants.

Consent for publication: not applicable 
Availability of data and materials: The datasets generated and/or analysed during the current study are not publicly available due to sensible contents but are available from the corresponding author on reasonable request.

Competing interests: The authors have no competing interests to declare.

Funding: This research received no specific grant from any funding agency in the public, commercial, or non-profit sectors.

Authors' contributions: FPB designed the study, collected the results, analyzed the data and drafted the manuscript. PS contributed to data collection. AMVL was responsible for the laboratory analysis. ST and CAG revised the protocol of the study and the manuscript. The corresponding author attests that all listed authors meet authorship criteria and that no others meeting the criteria have been omitted. All authors have read and approved the submitted version of the manuscript.

Acknowledgements: The Authors would like to thank all health workers involved in the described screening activities.

\section{References}

1. CDC. About Chickenpox. Available on: https://www.cdc.gov/chickenpox/about/index.html. Last accessed: 22 November 2020.

2. Marin M, Guris D, Chaves SS, et al. Prevention of Varicella. Recommendations of the Advisory Committee on Immunization Practices (ACIP). MMWR Morb Mortal Wkly Rep. 2007;56(RR04);1-40

3. WHO. Varicella. Vaccine-Preventable Diseases. Surveillance Standards. Available on: https://www.who.int/immunization/monitoring_surveillance/burden/vpd/WHO_SurveillanceVaccinePreventable_22_Varicella_R1.pdf? ua=1. Last accessed: 24 November 2020.

4. WHO. Immunization, Vaccines and Biologicals. Varicella. Available on: https://www.who.int/immunization/diseases/varicella/en/. Last accessed: 22 November 2020.

5. CDC. Monitoring the Impact of Varicella Vaccination. Available on: https://www.cdc.gov/chickenpox/surveillance/monitoringvaricella.html. Last accessed: 20 November 2020.

6. CDC. Impact of the Varicella Vaccination Program. Available on: https://www.cdc.gov/chickenpox/hcp/index.html\#vaccinationimpact. Last accessed: 15 November 2020.

7. CDC. Varicella Vaccine Recommendations. Available on: https://www.cdc.gov/vaccines/vpd/varicella/hcp/recommendations.html. Last accessed: 24 November 2020.

8. CDC. About the Varicella Vaccines. Available on: https://www.cdc.gov/vaccines/vpd/varicella/hcp/about-vaccine.html. Last accessed: 28 November 2020.

9. Stefanizzi P, De Nitto S, Patano F, Bianchi FP, Ferorelli D, Stella P, Ancona D, Bavaro V, Tafuri S. Post-marketing surveillance of adverse events following measles, mumps, rubella and varicella (MMRV) vaccine: retrospecive study in apulia region (ITALY), 2009-2017. Hum Vaccin Immunother. 2020 Aug 2;16(8):1875-1883.

10. Stefanizzi P, Stella P, Ancona D, Malcangi KN, Bianchi FP, De Nitto S, Ferorelli D, Germinario CA, Tafuri S. Adverse Events Following Measles-Mumps-Rubella-Varicella Vaccination and the Case of Seizures: A Post Marketing Active Surveillance in Puglia Italian Region, 2017-2018. Vaccines (Basel). 2019 Oct 7;7(4):140.

11. Italian Ministry of Health. Decree-Law 07 June 2017, n. 73. Urgent provisions on vaccination prevention. G.U. General Serie, $\mathrm{n} .130$ of 07 June 2017. Available on: http://www.trovanorme.salute.gov.it/norme/dettaglioAtto?id=59548. Last accessed on 25 november 2020.

12. Italian Ministry of Health. National Plan of Vaccinal Prevention (PNPV) 2017-2019. Available on: http://www.salute.gov.it/imgs/C_17_pubblicazioni_2571_allegato.pdf. Last accessed on 29 November 2020.

13. Balbi O, Baldi S, Rizza S, Pietroiusti A, Perrone S, Coppeta L. Seroprevalence survey for Varicella among healthcare workers and medical students in Italy. Hum Vaccin Immunother. 2020 Jul 9:1-5.

14. Coppeta L, D'Alessandro I, Pietroiusti A, Somma G, Balbi O, lannuzzi I, Magrini A. Seroprevalence for vaccine-preventable diseases among Italian healthcare workers. Hum Vaccin Immunother. 2020 Oct 5:1-5.

15. Trombetta CM, Montomoli E, Viviani S, Coluccio R, Marchi S. Evaluation of Varicella Immunity during Pregnancy in Apulia Region, Southern Italy. Vaccines (Basel). 2020 May 10;8(2):214.

16. Tafuri S, Gallone MS, Cappelli MG, Gallone MF, Larocca AM, Germinario C. A seroprevalence survey on varicella among adults in the vaccination era in Apulia (Italy). Vaccine. 2014 Nov 12;32(48):6544-7. 
17. Tafuri S, Martinelli D, De Palma M, Germinario C, Prato R. Report of varicella outbreak in a low vaccination coverage group of otherwise healthy children in Italy: the role of breakthrough and the need of a second dose of vaccine. Vaccine. 2010 Feb 10;28(6):1594-7

18. Bianchi FP, Gallone MS, Gallone MF, Larocca AMV, Vimercati L, Quarto M, Tafuri S. HBV seroprevalence after 25 years of universal mass vaccination and management of non-responders to the anti-Hepatitis $B$ vaccine: an Italian study among medical students. $\mathrm{J}$ Viral Hepat. 2018.

19. DiaSorin. The Diagnostic Specialist. LIAISON® VZV IgG. The fully automated solution for antibody detection. Available on: https://www.diasorin.com/sites/default/files/allegati_prodotti/ese_scheda_vzv_rev_02_low.pdf. Last accessed on 01 December 2020.

20. Cabrillo College. Clinical Compliance Basics. Health Screening. Varicella. Available on: https://www.cabrillo.edu/services/health/clinical-compliance/clinical-varicella.html. Last accessed 04 November 2020.

21. Bianchi FP, Stefanizzi P, De Nitto S, Larocca AMV, Germinario C, Tafuri S. Long-term immunogenicity of Measles vaccine: an Italian retrospective cohort study. J Infect Dis. 2019;jiz508.

22. Morris GP. Understanding sex-related differences in immune responses. Science Translational Medicine. 2020 Jul; Vol. 12, Issue 554, eabd3631.

23. Ortona E, Pierdominici M, Rider V. Editorial: Sex Hormones and Gender Differences in Immune Responses. Front Immunol. 2019;10:1076.

24. Bianchi FP, De Nitto S, Stefanizzi P, Larocca AMV, Germinario CA, Tafuri S. Immunity to rubella: an Italian retrospective cohort study. BMC Public Health. 2019;19(1):1490.

25. Bianchi FP, De Nitto S, Stefanizzi P, Larocca AMV, Germinario CA, Tafuri S. Long time persistence of antibodies against Mumps in fully MMR immunized young adults: an Italian retrospective cohort study. Hum Vaccin Immunother. 2020 Mar 18:1-7.

26. Behrman A, Lopez AS, Chaves SS, Watson BM, Schmid DS. Varicella immunity in vaccinated healthcare workers. J Clin Virol. 2013 Jun;57(2):109-14.

27. Trevisan A, Nicolli A, De Nuzzo D, Lago L, Artuso E, Maso S. Varicella seroepidemiology and immunization in a cohort of future healthcare workers in the pre-vaccination era. Int J Infect Dis. 2020 Jul;96:228-232.

28. Seward JF, Zhang JX, Maupin TJ, Mascola L, Jumaan AO. Contagiousness of varicella in vaccinated cases: a household contact study. JAMA. 2004 Aug 11;292(6):704-8.

29. Ampofo K, Saiman L, LaRussa P, Steinberg S, Annunziato P, Gershon A. Persistence of immunity to live attenuated varicella vaccine in healthy adults. Clin Infect Dis. 2002 Mar 15;34(6):774-9.

30. Leung J, Broder KR, Marin M. Severe varicella in persons vaccinated with varicella vaccine (breakthrough varicella): a systematic literature review. Expert Rev Vaccines. 2017 Apr;16(4):391-400.

31. Ludwig B, Kraus FB, Allwinn R, Keim S, Doerr HW, Buxbaum S. Loss of varicella zoster virus antibodies despite detectable cell mediated immunity after vaccination. Infection. 2006 Aug;34(4):222-6.

32. Tourtelot E, Quataert S, Glantz JC, Perlis L, Muthukrishnan G, Mosmann T. Women who received varicella vaccine versus natural infection have different long-term T cell immunity but similar antibody levels. Vaccine. 2020 Feb 11;38(7):1581-1585.

33. CDC. Shingles (Herpes Zoster). Available on: https://www.cdc.gov/shingles/hcp/clinical-overview.html. Last accessed on 03 december 2020.

\section{Figures}




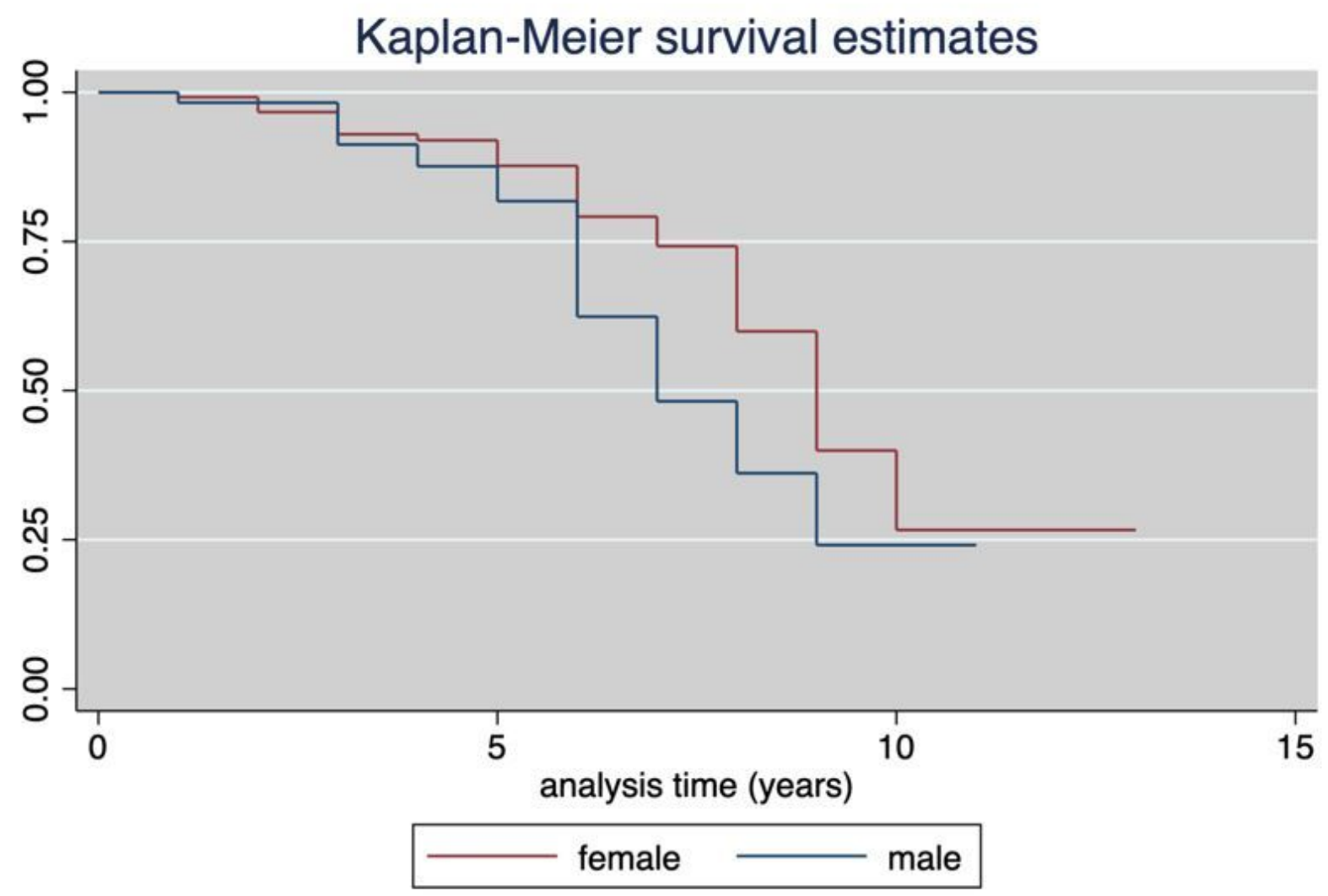

$\mathrm{p}=0.021$

Figure 1

Kaplan-Meier estimates of protective antibody survival (PAS) in males vs. females 